\title{
Моделирование образования каскада смещений и переходных ионизационных процессов в кремниевых полупроводниковых структурах при нейтронном воздействии
}

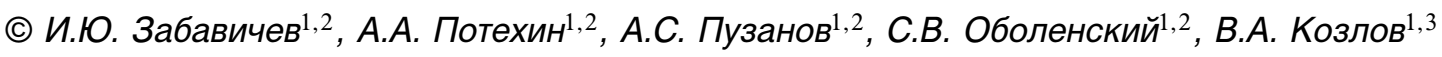 \\ ${ }^{1}$ Нижегородский государственный университет им. Н.И. Лобачевского, \\ 603950 Нижний Новгород, Россия \\ 2 Филиал „Российского федерального ядерного Всероссийского научно-исследовательского института \\ экспериментальной физики“ „Научно-исследовательский институт измерительных систем им. Ю.Е. Седакова“, \\ 603950 Нижний Новгород, Россия \\ ${ }^{3}$ Институт фризики микроструктур Российской академии наук, \\ 603087 Нижний Новгород, Россия \\ E-mail: zabavichev.rf@gmail.com
}

Поступила в Редакцию 24 апреля 2019 г.

В окончательной редакции 29 апреля 2019 г.

Принята к публикации 29 апреля 2019 г.

\begin{abstract}
При помощи метода молекулярной динамики проведено моделирование формирования разупорядоченной области дефектов в объемном кремнии для различных энергий первичного атома отдачи. Рассчитаны изменения объема и числа радиационных дефектов в кластере в процессе его формирования. Теоретически получены скорости генерации неравновесных носителей заряда и амплитудно-временны́е зависимости импульсов ионизационных токов в тестовых диодах Шоттки гипервысоких частот.
\end{abstract}

Ключевые слова: метод молекулярной динамики, кластер радиационных дефектов, высокопроизводительные вычисления.

DOI: $10.21883 /$ FTP.2019.09.48139.23

\section{1. Введение}

Основной тенденцией развития микро- и наноэлектроники является уменьшение размеров рабочих областей полупроводниковых структур [1], что в конечном счете обусловлено улучшением параметров интегральных схем и дискретных элементов (быстродействия и производительности, объема памяти, предельных рабочих частот, усиливаемой мощности, уровня шумов и т.д.) при сохранении или даже уменьшении стоимости их разработки и производства. Аналогичные процессы (с некоторой задержкой во времени) идут и в области разработки специализированной элементной базы, предназначенной для применения в военных и космических системах.

Перенос носителей заряда в нанометровых объемных [2] и квантово-размерных [3] структурах при радиационном воздействии имеет ряд особенностей, обусловленных усилением влияния неоднородности дефектообразования [4] и ионизации, а также возможностью полного перекрытия канала полевого транзистора или базы биполярного транзистора [5] одиночным кластером радиационных дефектов.

Экспериментально процесс формирования кластера радиационных дефектов на начальной стадии еще не изучен [6], хотя существуют предпосылки для решения данной задачи на основе анализа спектра излучения в терагерцовом диапазоне частот [7] или при помощи фемтосекундной съемки в режиме реального времени [8]. Таким образом, теоретическое рассмотрение процесса формирования разупорядоченной области радиационных дефектов в настоящее время остается единственной возможностью и представляет значительный интерес для построения математических моделей реакции субмикронных полупроводниковых приборов на радиационное воздействие.

В настоящее время существует широкий спектр методов моделирования развития каскадов атомных столкновений в веществе [6], которые можно разделить на 4 класса: квантово-механические методы „из первых принципов“, методы классической молекулярной динамики, методы парных столкновений на базе алгоритмов Монте-Карло и континуальные методы, основанные на законах тепломассопереноса в сплошной среде. В основе квантово-механических методов лежит численное решение многочастичного уравнения Шредингера. В силу вычислительной сложности данный метод ограничен системами, состоящими из нескольких сотен атомов, чья динамика моделируется в течение нескольких фемтосекунд [9]. Поэтому в настоящее время квантовомеханический метод на практике для моделирования развития каскадов атомных столкновений в веществе не применяется.

Метод классической молекулярной динамики, в котором временна́я эволюция системы взаимодействующих атомов отслеживается интегрированием их уравнений движения, впервые применен для анализа дефектообразования в материалах при радиационном воздействии в работе $[10]$. В данной работе помимо расчета эволюции точечных дефектов: их кластеризации в устойчи- 
вые скопления, а также рекомбинации части вакансий и межузельных атомов рассмотрена задача ионизации микрообъема первичным атомом отдачи и генерации импульсов тока в полупроводниковых структурах.

\section{2. Методика моделирования}

Упругое взаимодействие быстрых нейтронов с энергией свыше 0.1 МэВ с полупроводниковыми структурами приводит к тому, что частица выбивает из кристаллической решетки один из атомов вещества [11], называемый первичным атомом отдачи. Этот атом с достаточно высокой энергией (до сотен кэВ), тормозясь в среде за счет потерь на возбуждение и ионизацию электронной подсистемы кристалла, периодически упруго рассеивается на атомах среды, что в свою очередь выбивает их из занимаемого положения, в результате чего каскад смещений разветвляется наподобие дерева. Таким образом, в результате взаимодействия нейтрона со средой образуется импульс ионизационного тока, а движение носителей заряда в полупроводниковой структуре меняется из-за дополнительного рассеяния на формирующемся кластере радиационных дефектов. Ввиду существенно разных энергий и масс атомов отдачи и носителей заряда процессы формирования кластера радиационных дефектов и генерации импульса ионизационного тока можно рассматривать независимо.

\section{1. Методика расчета образования кластера радиационных дефектов}

Для моделирования процесса образования кластера радиационных дефектов использовался пакет прикладных программ LAMMPS [12]. В качестве межатомного потенциала взаимодействия был выбран потенциал Стилленгера-Вибера (Stillinger-Weber) [13]

$$
\begin{gathered}
U(r)=\sum_{i} \sum_{j>i} U_{2}\left(r_{i j}\right)+\sum_{i} \sum_{j \neq i} \sum_{k \neq j \neq i} U_{3}\left(r_{i j}, r_{i k}, \theta_{i j k}\right), \\
U_{2}\left(r_{i j}\right)=\left\{\begin{array}{lc}
A_{i j} \varepsilon_{i j}\left[B_{i j}\left(\frac{\sigma_{i j}}{r_{i j}}\right) p_{i j}-\left(\frac{\sigma_{i j}}{r_{i j}}\right) q_{i j}\right] \\
\times \exp \left(\frac{\sigma_{i j}}{r_{i j}-a_{i j} \sigma_{i j}}\right), & r_{i j} \leq a_{i j} \sigma_{i j} \\
0, & r_{i j} \geq a_{i j} \sigma_{i j}
\end{array}\right. \\
U_{3}\left(r_{i j}, r_{i k}, \theta_{i j k}\right)=\lambda_{i j k} \varepsilon_{i j k}\left[\cos \left(\theta_{i j k}\right)+\cos \left(\theta_{0 i j k}\right)\right]^{2} \\
\quad \times \exp \left(\frac{\gamma_{i j} \sigma_{i j}}{r_{i j}-a_{i j} \sigma_{i j}}\right) \exp \left(\frac{\gamma_{i k} \sigma_{i k}}{r_{i k}-a_{i k} \sigma_{i k}}\right),
\end{gathered}
$$

где $A, B, p, q, a, \lambda$ и $\gamma-$ безразмерные параметры модели; $\sigma$ - расстояние отсечки, $\varepsilon-$ размерный коэффициент. Значения параметров модели представлены в табл. 1. Использование данного потенциала обусловлено тем, что полученные с его помощью данные хорошо согласуются с теоретическими результатами моделирования и экспериментом [14].
Таблица 1. Значения параметров потенциала СтилленгераВибера [13]

\begin{tabular}{c|c}
\hline Название параметра & Значение \\
\hline$A$ & 7.049 \\
$B$ & 0.602 \\
$p$ & 4 \\
$q$ & 0 \\
$a$ & 1.8 \\
$\lambda$ & 21 \\
$\gamma$ & 1.2 \\
$\sigma$, нм & 0.209 \\
$\varepsilon$, эВ & 2.168
\end{tabular}

Таблица 2. Значения параметров двухтемпературной модели [14]

\begin{tabular}{c|c}
\hline Название параметра & Значение \\
\hline$C_{e}$, эВ $/\left(e^{-} \cdot \mathrm{K}\right)$ & $5.11511 \cdot 10^{-6}$ \\
$\rho_{e}, E^{-} / \mathrm{A}^{3}$ & 0.05 \\
$D_{e}, \mathrm{~A}^{2} / \Pi \mathrm{c}$ & 20000 \\
$\gamma_{p}, \Gamma /($ моль $\cdot \Pi \mathrm{c})$ & 1.741 \\
$\gamma_{s}, \Gamma /($ моль $\cdot$ пс $)$ & 39.23 \\
$v_{0}, \mathrm{~A} / \Pi \mathrm{c}$ & 79.76
\end{tabular}

Для учета электронных потерь движущихся атомов использовалась двухтемпературная модель [15], которая основана на уравнении тепловой диффузии

$$
C_{e} \rho_{e} \frac{\partial T_{e}}{\partial t}=\nabla\left(K_{e} \nabla T_{e}\right)-g_{p}\left(T_{e}-T_{a}\right)+g_{s} T_{a},
$$

где $C_{e}-$ теплоемкость электронного газа, $\rho_{e}-$ электронная плотность, $K_{e}-$ электронная проводимость, $g_{p}$ - коэффициент электрон-фононного взаимодействия, $g_{s}-$ коэффициент электронных потерь, $T_{a}$ и $T_{e}$ - температуры атомной и электронной подсистемы соответственно. Решив уравнение (2) относительно $T_{a}$ и $T_{e}$, можно вычислить обмен энергией между атомной и электронной подсистемами. Значения параметров модели, выбранные для проведения расчетов в данной работе, представлены в табл. 2 [14].

Размер расчетной области составил $500 \times 500 \times 500 \mathrm{~A}$ (8000000 атомов), в которой моделировалась эволюция разупорядоченной области атомных смещений в течение 100 пс, образованной первичным атомом отдачи кремния с начальной кинетической энергией 3, 12 и 50 кэВ. Для определения местоположения дефектов в моделируемой системе использовался метод, основанный на построении ячеек Вигнера-Зейтца. К полученному множеству точечных дефектов применялся алгоритм $k$-средних [16], выделяющий отдельные субкластеры в общем объеме каскада смещений, расстояние между которыми не превышает размеры области пространственного заряда в полупроводнике при заданном уровне легирования примесью. Каждый получившийся 
субкластер аппроксимировался эллипсоидом вращения в соответствии с алгоритмом [17] и вычислялся его объем.

\section{2. Методика расчета переходных ионизационных процессов в полупроводниковых структурах}

Для расчета реакции субмикронных полупроводниковых приборов на воздействие одиночных нейтронов использовалась модифицированная локально-неравновесная диффузионно-дрейфовая модель переноса носителей заряда, ранее предложенная в работе [18]. Радиационночувствительными параметрами модели являются времена жизни, подвижности и коэффициенты диффузии электронов и дырок, значения которых уменьшаются с ростом флюенса или дозы дефектообразующих излучений. Учет ионизационной компоненты осуществляется через пространственно-временно́е изменение скорости генерации неравновесных носителей заряда.

Точный расчет пространственно-временно́го распределения неравновесных носителей заряда в процессе прохождения заряженной частицы через вещество представляет собой отдельную, до конца не решенную задачу [19]. Традиционно для расчета переходных ионизационных процессов в полупроводниковых структурах при воздействии протонов, $\alpha$-частиц и тяжелых заряженных частиц космического пространства в системах автоматизированного проектирования изделий микро- и наноэлектроники используется представление скорости генерации неравновесных носителей заряда в виде произведения трех сомножителей $[20,21]$

$$
G(l, r, t)=G_{L E T}(l) R(r, l) T(t) .
$$

Здесь $G_{L E T}(l)$ - амплитудный коэффициент, определяемый величиной линейной передачи энергии вдоль трека частицы, $R(r, l)$ - нормированный пространственный множитель, описываемый экспоненциальной или гауссовой функциями, $T(t)-$ нормированный временно́й множитель, описываемый гауссовой функцией, $r-$ радиус, определяемый как перпендикулярное расстояние к треку, $l-$ расстояние вдоль трека. Трек частицы предполагается прямолинейным.

В данной работе использовалось более точное нормированное пространственное распределение неравновесных носителей заряда [19], которое в нормированном виде записывается как

$$
\begin{gathered}
R(r, l)=\frac{1}{2 \pi r_{c}^{2}}\left(1+\frac{1}{1+2 \ln \left(\frac{r_{p}}{r_{c}}\right)}\right), r<r_{c}, \\
R(r, l)=\frac{1}{2 \pi r}\left(1+\frac{1}{1+2 \ln \left(\frac{r_{p}}{r_{c}}\right)}\right), r_{c} \leq r \leq r_{p},
\end{gathered}
$$

где $r_{c}$ - радиус ядра, определяемый как отношение скорости частицы к плазмонной частоте (равной 16.7 эВ в случае кремния), $r_{p}$ - радиус оболочки, определяемый длиной пробега $\delta$-электронов.
Изменение энергии электронной подсистемы моделируемого объема определяется двумя конкурирующими механизмами: возбуждением электронной подсистемы атомами отдачи и релаксацией энергии в кристаллическую решетку. Таким образом, получаем уравнение

$$
\frac{d E_{e l}}{d t}=2 \pi E_{e h} \int_{0}^{\infty} \int_{0}^{\infty} r G(l, r, t) d r d l-\frac{E_{e l}}{\tau_{E}},
$$

где $E_{e l}$ - энергия электронной подсистемы за вычетом равновесной тепловой составляющей, $V-$ объем кластера радиационных дефектов, $\tau_{E}-$ среднее время релаксации энергии электронной подсистемы, полагавшееся равным 0.1 пс, $E_{e h}-$ средняя энергия, необходимая для образования электронно-дырочной пары (равная 3.6 эВ в случае кремния). Зависимость энергии электронной подсистемы от времени была получена при помощи расчета методом классической молекулярной динамики.

\section{3. Результаты расчетов и их обсуждение}

При помощи метода молекулярной динамики была рассчитана пространственно-временна́я эволюция области разупорядочения, образованная первичным атомом отдачи: временнб́е зависимости числа пар Френкеля (рис. 1), объема области структурных повреждений и ионизации (рис. 2), а также энергии, передаваемой в электронную подсистему (рис. 3). Полученные результаты были использованы для расчета скорости генерации неравновесных носителей заряда (рис. 4) и переходных ионизационных процессов в тестовой полупроводниковой структуре (рис. 5).

В процессе формирования кластера радиационных дефектов можно выделить 4 характерные стадии: баллистический участок (до 0.04 пс), в течение которого первичный атом отдачи движется практически без столкновений; участок термического расширения (от 0.04

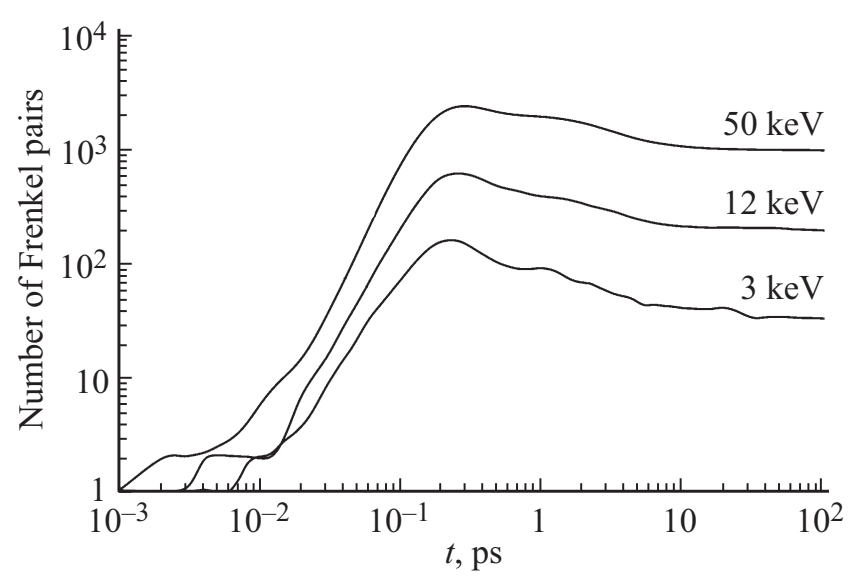

Рис. 1. Временна́я зависимость числа пар Френкеля для различных энергий первичного атома: 50, 12 и 3 кэВ. 


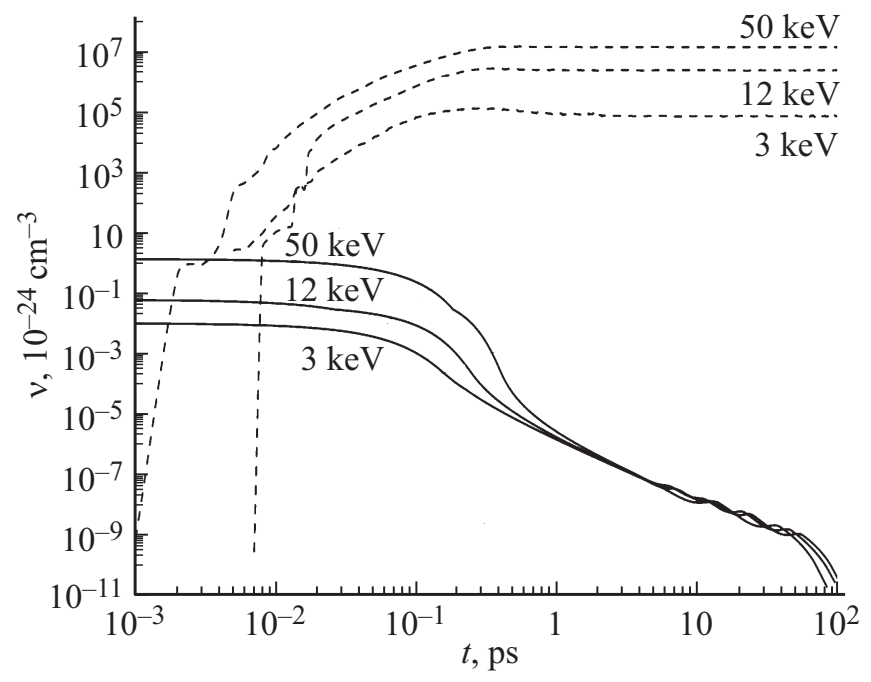

Рис. 2. Временна́я зависимость объема кластера радиационных дефектов (---) и объема области ионизации (-) для различных энергий первичного атома: 5, 12 и 3 кэВ.

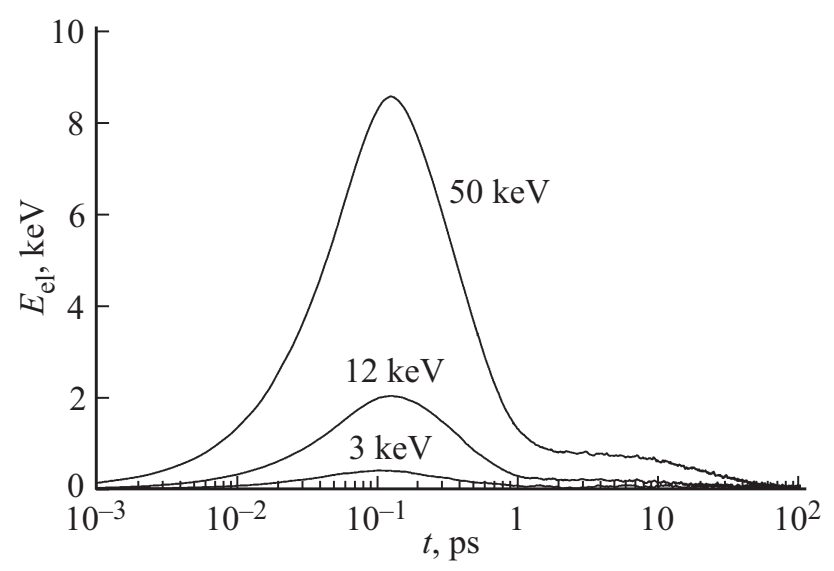

Рис. 3. Временна́я зависимость энергии электронной подсистемы для различных энергий первичного атома: 50, 12 и 3 кэВ.

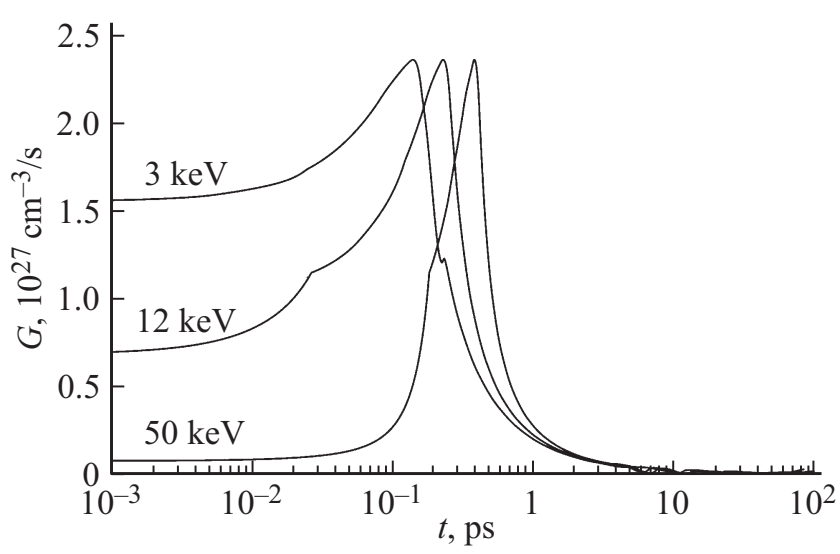

Рис. 4. Временна́я зависимость скорости генерации неравновесных носителей заряда для различных энергий первичного атома: 50,12 и 3 кэВ.

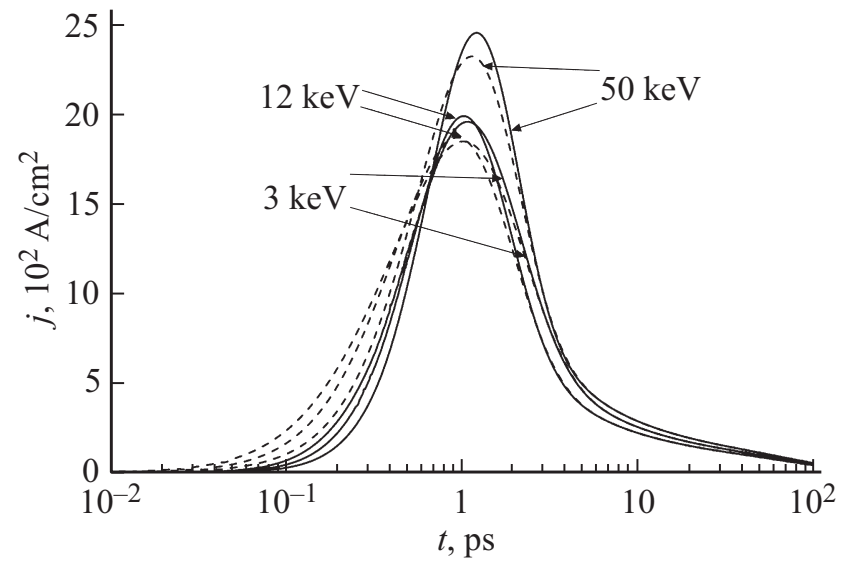

Рис. 5. Временна́я зависимость импульсов ионизационных токов, образованных первичными атомами отдачи различных энергий: 3,12 и 50 кэВ; (-) - локально-неравновесная диффузионно-дрейфовая модель; (---) - локально-равновесная диффузионно-дрейфовая модель.

до 0.2 пс), на протяжении которого число дефектов в кластере стремительно нарастает; стадия первичной стабилизации (от 0.2 до 0.5 пс), которая характеризуется резким уменьшением числа дефектов за счет рекомбинации близких пар Френкеля, и стадия быстрого отжига (от 0.5 пс), которая может длиться несколько наносекунд, сопровождаясь при этом плавным по сравнению со стадией первичной стабилизации уменьшением числа дефектов за счет отжига. Медленный темп рекомбинации дефектов на последней стадии объясняется термостимулированной диффузией дефектов в остывающем объемном полупроводнике, поэтому для адекватного описания последующего отжига необходим больший промежуток времени физического моделирования (от единиц секунд до нескольких часов). Стоит отметить, что объем кластера радиационных дефектов на стадии быстрого отжига практически не меняется, в то время как число дефектов в нем медленно уменьшается, что связано с рекомбинацией пар дефектов, расположенных в центре, а не на периферии разупорядоченной области.

В отличие от временно́й зависимости объема структурных повреждений зависимость объема области ионизации от времени носит монотонно убывающий характер, что обусловлено выносом носителей заряда внешним электрическим полем из области генерации. При этом зависимость энергии, передаваемой электронной подсистеме, от времени имеет максимум в диапазоне времен $0.1-0.2$ пс в зависимости от начальной энергии первичного атома отдачи, что обусловливает экстремальную форму зависимости скорости генерации неравновесных носителей заряда от времени. Время достижения максимума скорости генерации неравновесных носителей заряда увеличивается с ростом начальной энергии первичного атома отдачи и составляет: 0.14 пс для энергии 3 кэВ, 0.23 пс для энергии 12 кэВ и 0.38 пс для энергии 50 кэВ. При этом максимальная скорость 
генерации неравновесных носителей заряда не зависит от энергии в рассматриваемом диапазоне и составляет $2.4 \cdot 10^{27} \mathrm{~cm}^{-3} / \mathrm{c}$.

В качестве тестовой структуры был рассмотрен кремниевый диод Шоттки [18]. Концентрация примеси $n$-типа в рабочей области принималась равной $10^{17} \mathrm{~cm}^{-3}$, длина структуры 1 мкм, высота барьера Шоттки 0.55 В. На данную структуру в отсутствие внешнего приложенного напряжения моделировалось воздействие импульса ионизирующего излучения с параметрами, приведенными на рис. 4.

Результаты моделирования переходных ионизационных процессов приведены на рис. 5 для начальной энергии первичного атома отдачи 3,12 и 50 кэВ. Несмотря на одинаковую максимальную скорость генерации неравновесных носителей заряда, максимальная амплитуда импульса ионизационного тока зависит от начальной энергии первичного атома отдачи и составляет: $1.96 \kappa \mathrm{A} / \mathrm{cm}^{2}$ для 3 кэВ, 1.99 кА/см ${ }^{2}$ для 12 кэВ и 2.46 кА/см ${ }^{2}$ для 50 кэВ. Расчет по локально-равновесной диффузионно-дрейфовой модели дает заниженную оценку максимальной амплитуды ионизационного тока [18] и составляет: $1.85 \kappa \mathrm{A} / \mathrm{cm}^{2}$ для 3 кэВ, 1.87 кА/ $\mathrm{cm}^{2}$ для 12 кэВ и 2.32 кА/см ${ }^{2}$ для 50 кэВ. Это различие может оказаться важным для расчета одиночных событий в ячейках памяти интегральных схем, так как вероятность сбоя зависит не только от интегрального значения заряда, но и формы импульса ионизационного тока [22]. Время достижения максимальной амплитуды ионизационного тока лежит в пределах 0.94-1.21 пс, что существенно больше времени достижения максимальной скорости генерации неравновесных носителей заряда - 0.14-0.38 пс и слабо зависит от применяемой для расчета модели, что обусловлено большой длительностью переднего фронта импульса возбуждения по сравнению со средним временем между рассеяниями электронов и дырок, которое полагалось равным 0.1 пс.

\section{4. Заключение}

Результаты проведенных расчетов показывают возможность применения комплекса программ для расчета: формирования разупорядоченной области дефектов и ионизации микрообъема полупроводниковых структур первичными атомами отдачи при взаимодействии потоков нейтронов с веществом на основе метода классической молекулярной динамики и переходных ионизационных процессов при помощи локально-неравновесных моделей переноса носителей заряда.

Дальнейшее развитие предложенного подхода может быть связано с учетом переноса горячих носителей заряда, возникающих в треке первичного атома отдачи. Это потребует разработки локально-неравновесной квазигидродинамической модели переноса электронов и дырок в полупроводниковых структурах, а также методики расчета пространственно-временных распределений температуры неравновесных носителей заряда.

\section{Финансирование работы}

Исследование поддержано грантом Российского научного фонда (проект № 18-13-00066). Работа выполнена с использованием ресурсов суперкомпьютера „Лобачевский“ ННГУ им. Н.И. Лобачевского.

\section{Конфликт интересов}

Авторы заявляют, что у них нет конфликта интересов.

\section{Список литературы}

[1] International Roadmap for Devices and Systems. 2017 Edition, Copyright@2018 IEEE.

[2] С.В. Оболенский, Г.П. Павлов. ФТП, 29 (3), 413 (1995).

[3] Н.В. Демарина, С.В. Оболенский. ЖТФ, 72 (1), 66 (2002).

[4] А.С. Пузанов, С.В. Оболенский, В.А. Козлов. ФТП, 50 (12), 1706 (2016).

[5] И.Ю. Забавичев, А.А. Потехин, А.С. Пузанов, С.В. Оболенский, В.А. Козлов. ФТП, 51 (11), 1520 (2017).

[6] Б.А. Калин. Физическое материаловедение. Т. 4. Физические основы прочности. Радиационная физика твердого тела. Компьютерное моделирование (М., МИФИ, 2008) c. 231.

[7] А.С. Пузанов, С.В. Оболенский, В.А. Козлов, Е.В. Волкова, Д.Г. Павельев. ФТП, 49 (12), 1585 (2015).

[8] J. Liang, L. Zhu, L.V. Wang. Light: Sci. Appl., 7 (42), 1 (2018).

[9] D.D. Vvedensky. J. Phys.: Condens. Matter, 16, 1537 (2004).

[10] J.B. Gibson, A.N. Goland, M. Milgram, G.H. Vineyard. Phys. Rev., 120 (4), 1229 (1960).

[11] И.М. Дремин, О.В. Иванов, В.А. Нечитайло, Н.М. Соболевский, А.В. Субботин, В.П. Шевелько. ЖЭТФ, 125 (2), 362 (2004).

[12] S. Plimton. J. Comput. Phys., 117 (1), 1 (1995).

[13] F.H. Stillinger, T.A. Weber. Phys. Rev. B, 31 (8), 5262 (1985).

[14] A. Jay, M. Raine, N. Richard, N. Mousseau, V. Goiffon, A. Hemeryck, P. Magnan. IEEE Trans. Nucl. Sci., 64 (1), 141 (2017).

[15] D. Duffy, A. Rutherford. J. Phys.: Condens. Matter, 19, 016207 (2007).

[16] H. Steinhaus. (1956). Sur la division des corps materiels en parties. Bull. Acad. Polon. Sci., C1. III v. IV: 801-804.

[17] L.G. Khachiyan. Mathematics Operations Res., 21 (2), 307 (1996).

[18] А.С. Пузанов, С.В. Оболенский, В.А. Козлов. ФТП, 52 (11), 1295 (2018).

[19] Ф.Ф. Комаров. Успехи физ. наук, 187 (5), 465 (2017).

[20] Электронный pecypc https://www.synopsys.com

[21] Электронный pecypc https://www.silvaco.com

[22] К.О. Петросянц, И.А. Харитонов, Е.В. Орехов, Л.М. Самбурский, А.П. Ятманов, А.В. Воеводин. Сб. тр. 5-й Всеросс. науч.-техн. конф. „Проблемы разработки перспективных микро- и наноэлектронных систем - 2012“ (М., ФГБУ ИППМ РАН, 2012).

Редактор Г.А. Оганесян 


\section{Simulation of the formation of a cascade of displacements and transient ionization processes in silicon semiconductor structures under neutron exposure}

I.Yu. Zabavichev1,2, A.A. Potehin1,2, A.S. Puzanov1,2, S.V. Obolenskiy ${ }^{1,2}$, V.A. Kozlov ${ }^{3}$

${ }^{1}$ Lobachevski University of Nizhny Novgorod, 603950 Nizhny Novgorod, Russia

${ }^{2}$ Branch of Russian Federal Nuclear Center all-Russian Research Institute of Experimental Physics

"Sedakov Scientific Research Institute of Measurement Systems", 603950 Nizhny Novgorod, Russia

${ }^{3}$ Institute for Physics of Microstructure, Russian Academy of Sciences, 603087 Nizhny Novgorod, Russia

Abstract Using the method of molecular dynamics, we simulated the formation of a disordered region of defects in bulk silicon for different energies of the primary recoil atom. The changes in the volume and number of radiation defects in a cluster in the process of its formation are calculated. The rates of generation of nonequilibrium charge carriers and the amplitude - time dependences of the pulses of ionization currents in test Schottky diodes of hyperhigh frequencies are obtained theoretically. 